\title{
THE MICROTURBINE ROTATION BY NOT CIRCULAR LIGHT BEAM FORMED BY VORTEX AXICON
}

\author{
S.V. Ganchevskaya, R.V. Skidanov \\ Image Processing Systems Institute - Branch of the Federal Scientific Research Centre "Crys- \\ tallography and Photonics" of Russian Academy of Sciences, Samara, Russia \\ Samara National Research University, Samara, Russia
}

\begin{abstract}
The using possibility of the vortex light beam with topological charges 2 and 5 for the rotation of the micromechanical element (microturbine) was considered. The results of the full-scale experiment on the optical rotation of the special form microturbine were presented. We evaluated the force moment, acting the microturbine.
\end{abstract}

Keywords: microturbine, optical rotation, light beam.

Citation: Ganchevskaya SV, Skidanov RV. The microturbine rotation by not circular light beam formed by vortex axicon. CEUR Workshop Proceedings, 2016; 1638: 24-31. DOI: 10.18287/1613-0073-2016-1638-24-31

\section{Introduction}

The light beam with the orbital angular moment can be formed by the diffractive optical elements for a defined shape micromechanical component to optimize the process of the light energy transformation into mechanical energy. Such beams are widely used to rotate microscopic objects with the size of 0.1-10 microns, which is confirmed by the numerous publications. For example, the [1] work shows the optically controlled micromechanical system with the Archimedes helix as a mechanical drive in the form of the central sphere with three blades which were produced by two-photon polymerization. The laser beam is focused on the micro systems, the microhelical falls into a trapping site at the point of beam application and rotates simultaneously. In this article we research the dependence of the micro-rotor rotation speed with blades and without them, respectively equal to $1.9 \mathrm{~min}-1 / \mathrm{mW}$ and $13.5 \mathrm{~min}-1 / \mathrm{mW}$. The dependence of the rotation speed on thickness of the blade and central part of the helix was considered in the research [2]. However, it should be noted that the primary mechanism for the increase of the microscopic objects rotation speed is the power increase of the light beam, but the three-dimensional structures based on polymers do not have sufficient beam resistance. The metallized, cross-shaped rotors (diameter of 
$40 \mu \mathrm{m})$ was developed for the microdrive motors by scanning of the laser low power beam to solve this problem in the research [3]. The analysis of the light pressure exerted on the blade was carried out, and its results show that the oblique blade can generate more rotational moment than the horizontal blade due to the reflection of the incoming Gaussian beam. The metallized microturbine (diameter of $60 \mu \mathrm{m}$, height 6 $\mu \mathrm{m})$ which is effectively used to create the movement of the fluid stream was also developed in the research [4]. Based on the two-photon polymerization efforts for miniaturization of the diffractive optical element (DOE) were efforted to form the light vortex beams [5]. As a result, the DOE for the formation of a vortex beam was comparable in the scale with the rotated microscopic objects (diameter of $9 \mu \mathrm{m}$, at a resolution of $0.3 \mu \mathrm{m})$.

However, the number of the researches on the use of light vortex beams with microscopic objects in a rarefied medium (air or vacuum), is not enough, due to the significant technical difficulties during the experiments realization. Therefore, it is important to develop a system where the light beams and micromechanical elements will be designed to each other and are optimized for the operation in air or vacuum. But with the use of the diffractive optical elements we are able to realize the system where the operating micromechanical element will be illuminated by the light beam with the orbital angular moment, which will be formed to fit the shape of the microscopic objects, so that the rotation moment transmission can occur due to the light pressure as a result of the light reflection from the surface. As the result, it is possible to minimize the light absorption by the microscopic object, and consequently its thermal effect.

One the way to minimize the thermal effect is the distribution of the light beam intensity by the maximum area. Such vortex beams exist, for example, Bessel beams [6].

However, Bessel beams are not very suitable, because the intensity of rings sharply decreases with the number increase. It is easy to use the superposition of the vortex beams that due to the gradual increase of the topological charge will provide the change of the vortex beam size to realize the estimated distribution of the intensity [7]. These beams allow different types of the impact on the microscopic object. The research [7] shows in details the different variations of the topological charge and the estimated effect of these beams on the microscopic objects. Furthermore, despite the vortex nature of beams, this effect does not necessarily lead to the rotation of the microscopic object as the research [8] shows the linear trap with anisotropic behavior on the basis of these vortex superpositions. However, if the condition contained in the research [9] is met, any beam obtained by the method described in the research [7], can be used to rotate microscopic objects. In this case, the configuration of a required intensity distribution can also be realized automatically [10]. In this research we consider one of the superpositions, which is ideal to rotate the microscopic objects of the special form, obtained by the method of the research $[7,10]$. 


\section{Formation of the superposition of light fields by vortex axicons for the microturbine rotation}

To rotate the microturbine, it was decided to use one of the beams represented in the research [7], which meets the requirements [9]. To select the light beam, we were guided by the following considerations:

- the tilt of the wavefront at each point of the beam must be perpendicular to the surface of the microturbine blade;

- the beam must satisfy the requirements of the intension distribution in the polar angle[8].

The Figure 1 shows the phase function of the DOE (Fig. 1a), and formed intensity distribution (Fig. 1b) and the beam phase (Fig. 1c).

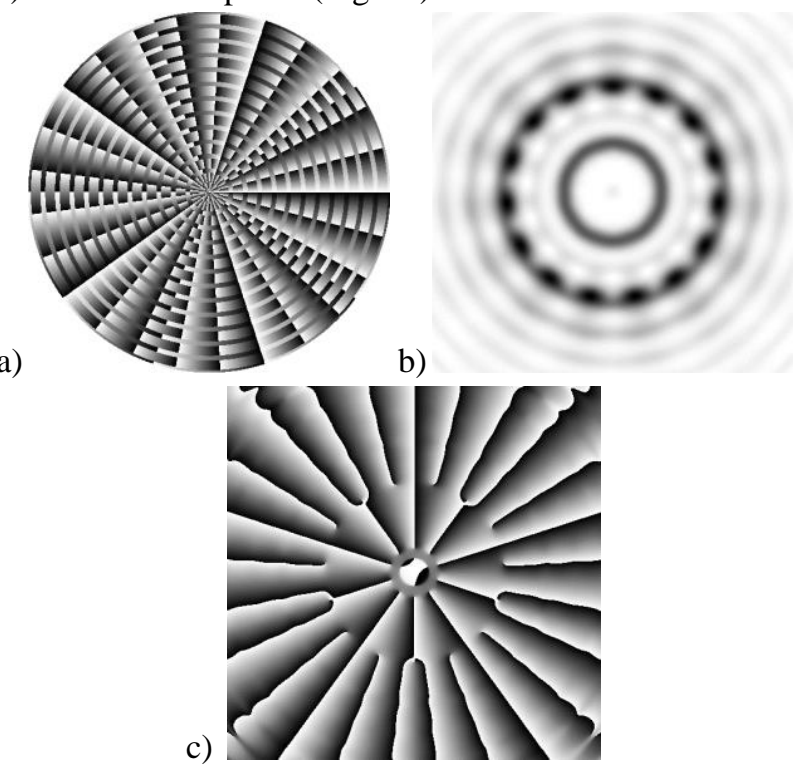

Fig. 1. Phase function of the DOE (a), the intensity distribution in the far field of diffraction (b), the phase of the beam (c)

Initially, this beam is assumed to rotate the microturbines from an opaque material, however, this beam enables to rotate the set of the polystyrene micropatricles, and as will be shown below, the beam successfully rotates the transparent microturbines.

To minimize thermal effect on the microturbine it is necessary to form the light beam in the way that the intensity of the beam has been distributed over the maximum area and the beam size was approximately comparable to the size of the microturbine (Fig. 2). Also, for efficient transmission of the rotation moment from the vortex beam to the microturbine the following requirement should be satisfied: the intension, acting 
the microturbine blade, is on the same level, according to its position, it is achieved on the basis of the intension distribution in the polar angle [9].

$$
I_{S}(\varphi)=\int_{0}^{R} I(\mathrm{r}, \varphi) \mathrm{dr} \approx \text { const }
$$

where $I(r, \varphi)$ is the intensity of the vortex beam in polar coordinates centered on the beam axis, $I_{s}$ is the total intensity of the beam at the current narrow sector.

a)

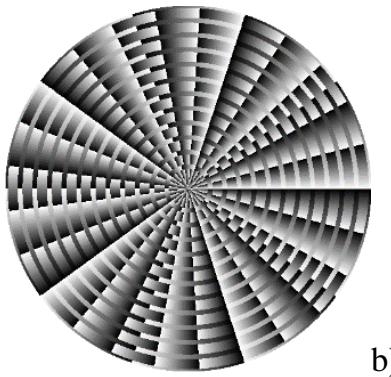

b)

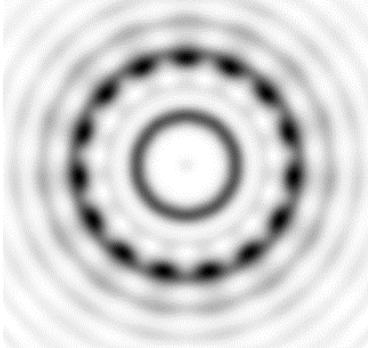

Fig. 2. The phase function of the DOE (a), the beam intensity distribution in the far field of the diffraction (b), the phase of the beam in the far field of the diffraction (c).

The requirement is satisfied for the beam shown in the Figure 2b. The Figure 3 shows the $I_{s}$ distribution according to the polar angle.

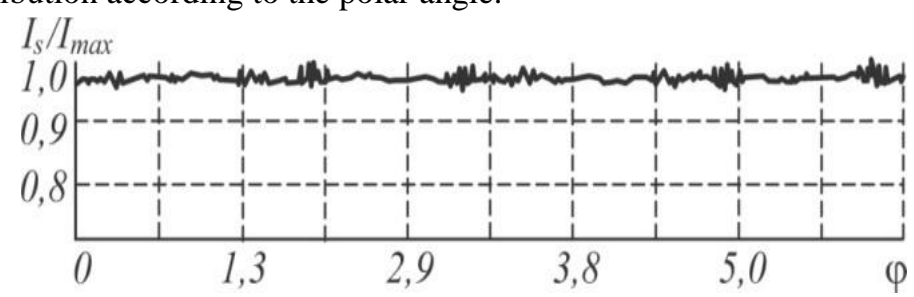

Fig. 3. The total intensity distribution of the generated beam

The deviation of the $I_{s}$ from the average value does not exceed 0.05 . 


\section{Experiments on the rotation of the microturbine}

The microscopic objects of the special form (microturbines) were designed in order to realize the rotation experiment. The circular laser work station (CLWS-2014) was used for this purpose. The optical setup, which scheme shown in the Figure 2, was used to form the complicated vortex beams and to focus them on the microturbines.

The laser with a wavelength of $532 \mathrm{~nm}$ and a maximum power of $4 \mathrm{~W}$ was used as the radiation source. The laser beam input is realized from below to reduce the friction which is increased when the laser beam is focused on the top and presses the particles to the substrate by the light pressure. The beam was increased to a diameter of about $2.3 \mathrm{~mm}$ by the collimator, and then was limited to $2 \mathrm{~mm}$ by the iris aperture to match the size of the beam to the size of the DOE. The microlens 16x were used to focus the beam and to form the operation area image. The microturbines were placed in a cell with water and salt was added to increase its density. Because the density of the resist material whereof the microturbine was produced is $1200 \mathrm{~kg} / \mathrm{m}^{3}$, that is much higher than the water density, the salt addition reduces the microturbine weight and consequently decreases friction.

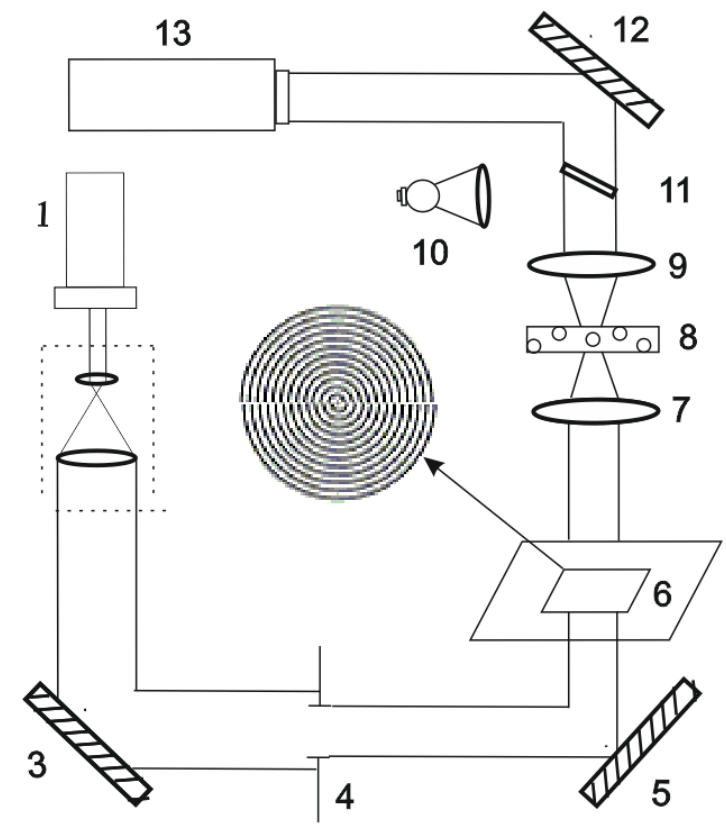

Fig. 4. The optical scheme of the microturbines rotation setup installation. The optical scheme uses the keys: 1 - the laser with a wavelength of $532 \mathrm{~nm}$ and a maximum power of $4 \mathrm{~W} ; 2$ collimator; 3, 5, 12 - rotating mirror; 4 - the aperture; 6 - axicon; 7 - a focusing microlens $(\times$ 16); 8 - substrate with microturbines; 9 - imaging microlens ( $\times 16)$; 10 - spotlight; 11 - halftransmitting rotating mirror; 13 - charge-coupled devices camera (CCD). 
The operation area was illuminated with white light from a standard illuminator. The recoding was carried out by the CCD camera and the spectral filter was placed in front of it. The Figure 3 shows the stages of the microturbine rotation.

a)

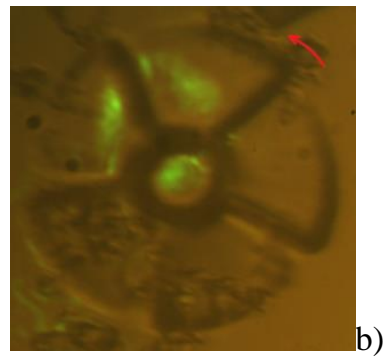

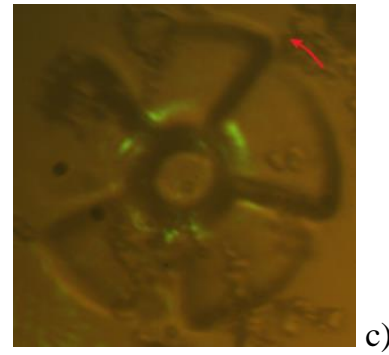

c)

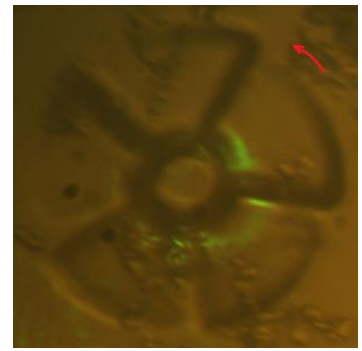

d)

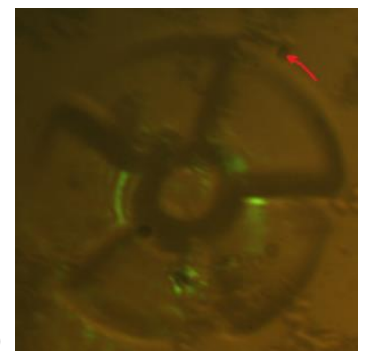

Fig. 5. The stages of the polymer microturbine rotation with the interval of $2 \mathrm{~s}$ in the beam with the topological charges 5, 2, 5

The Figure 5 shows that the rotation of the microturbine with the diameter of $100 \mu \mathrm{m}$ was realized by the focused light beam. We calculate the rotating speed of the microturbine and the moment of the friction, acted it, using the first and the last image. We find the coordinates of the blade in position before and after rotation. To calculate the friction moment we use the formula for the moment of friction acting the wheel of the terminating radius[11]:

$M=-1,94 \cdot R^{4} \rho \sqrt{v \Omega^{3}}$,

where $\mathrm{R}$ is a radius of the microturbine, $\rho$ is the water density, $v$ - viscosity factor at $20^{\circ} \mathrm{C}, \Omega$ - the rotating speed.

The rotating speed of the microturbine was $0.056 \mathrm{c}^{-1}$, and the moment of the rotating intensity acting it is $2.87 \mathrm{fmN} \cdot m$. This enables the evaluation of the total intensity exerted on the microturbine by the beam - it is about $100 \mathrm{pN}$.

The weight of the turbine is decreased with adding salt, which is resulted in microturbine rotation. However, there are some difficulties: before each intake of the drop it is necessary to heat the solution in the water bath for several minutes, but even in this Information Technology and Nanotechnology (ITNT-2016) 
case the salt is not completely dissolved - figures show salt crystals, which are the obstacle for the microturbine rotation. Therefore, another method based on the highfrequency vibration was used to minimize the friction. The special device for the creation of the high-frequency mechanical vibrations was added to the setup. The experiment was realized using light traps, designed for the microturbine rotation (Fig. 2b).

a)
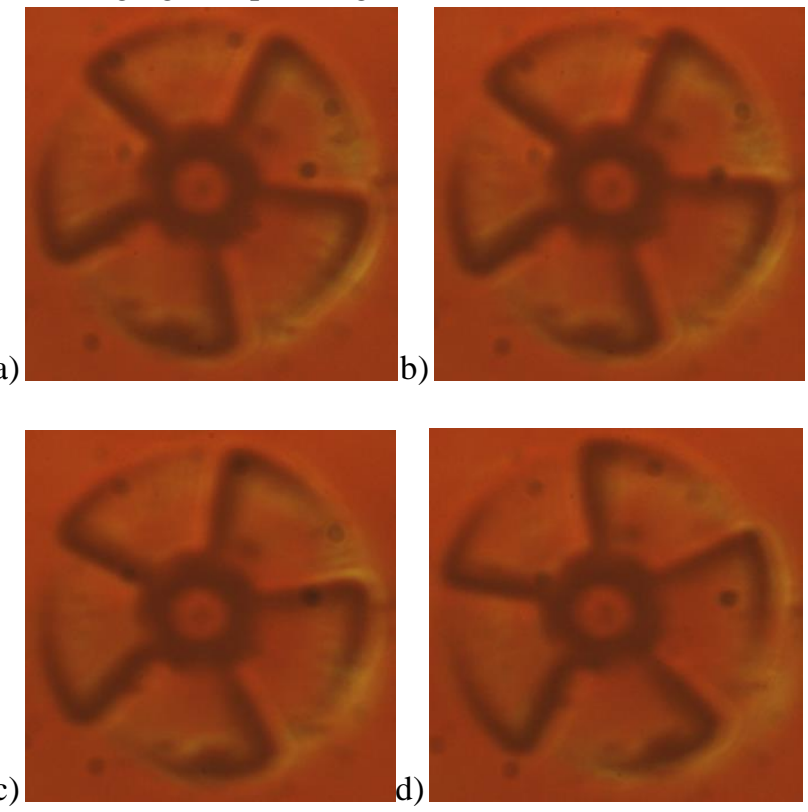

Fig. 6. Stages of microturbine rotation, taken with an interval of 2 second

The microturbine rotation speed at high-frequency vibrations is that exceeds the speed of microturbine rotation in salt brine using a beam, shown in the Figure $1 \mathrm{~b}$. It proves the effectiveness of the proposed method to the experiment realization.

\section{Conclusion}

The complex vortex beam with topological charges 2 and 5, in spite of the noncircular structure that allows the microturbine, that matches in the form with its wavefront, to be rotated. It is necessary to reduce the weight of the microturbines by increasing the fluid density for the successful experiments with such large objects as the microturbine (diameter $100 \mu \mathrm{m}$ ). The intensity moment acting the microturbine was about $3 \mathrm{fmN}$, which is quite a high value within the parameters of the optical circuit used in the experiment. We proved the perspective of the vibration use in the rotation experiments. 


\section{Acknowledgements}

The work was funded RSF grant 14-19-00114.

\section{References}

1. Liu Yi-J, Lee Yi-H, Lin Yu-Sh, Tsou Ch, Baldeck PL, Lin Ch-L. Optically Driven Mobile Integrated Micro-Tools for a Lab-on-a-Chip. Actuators, 2013; 2(2): 19-26.

2. Lina Ch-L, Lina Yu-Sh, Baldeck P. Rotational Efficiency of Photo-Driven Archimedes Screws for Micropumps. Micromachines, 2015; 6: 674-683.

3. Ikegami1 T, Ozawa1 R, Stocker MP, Fourkas JT, Maruo S. Development of OpticallyDriven Metallic Microrotors Using Two-Photon Microfabrication. JLMN-Journal of Laser Micro/Nanoengineering, 2013; 8(1): 6-10.

4. Ikegami1 T, Ozawa1 R, Stocker MP, Fourkas JT, Maruo S. Active micromixer using a metallized microturbine driven by an ultra-low power laser. 16th International Conference on Miniaturized Systems for Chemistry and Life Sciences, 2012; 5: 139.

5. Knoener G, Parkin S, Nieminen TA, Loke VLY, Heckenberg NR, Rubinsztein-Dunlop H. Integrated optomechanical microelements. Optics Express, 2007; 15(9): 5521-5530.

6. Skidanov RV, Ganchevskaya SV. Formation of Bessel beams by vortex axicon. Computer Optics, 2014; 38(3): 463-468.

7. Skidanov RV, Ganchevskaya SV. Diffractive optical elements for the formation of combinations of vortex beams in the problrm manipulation of microobjects. Computer Optics, 2014; 38(1): 65-71.

8. Skidanov RV, Porfirev SV, Ganchevskaya SV. Manipulation of micro-objects using linear traps generated by vortex axicons. Computer Optics, 2014; 38(4): 717-721.

9. Morozov AA, Skidanov RV. Complex vortex beams for of rotation of micromechanical elements. Computer Optics, 2013; 37(1): 68-75.

10. Skidanov RV, Ganchevskaya SV. The algorithm of calculation of the DOE for forming the configuration of the light traps. Computer Optics, 2015; 39(2): 181-186. DOI: 10.18287/0134-2452-2015-39-2-181-186

11. Landau LD, Lifshitz EM. Hydrodynamics, 3rd edition. Nauka, Moscow, 1986; 736. 